\title{
Secondary Peritonitis in Peritoneal Dialysis: A Case Report and Review of Literature
}

\author{
Muhammad Imran Khan a , Firas Ahmed Adeel ${ }^{\mathrm{b}}$, Faraz Khan Luni ${ }^{\mathrm{b}}$, Abdur Rahman Khan ${ }^{\mathrm{b}}$, \\ Deepak Malhotra ${ }^{\mathrm{c}}$, Joan Duggan ${ }^{\mathrm{d}, \mathrm{e}}$
}

\begin{abstract}
A 41-year-old female with Down's syndrome on peritoneal dialysis (PD) presented with PD-related peritonitis which was not responding to guideline-directed antimicrobial therapy. Computed tomography scan revealed air in the peritoneal cavity initially suspected to be secondary to her PD. Multiple enteric bacteria were identified in the PD fluid which raised suspicion for perforation. A perforated diverticulum was eventually diagnosed with exploratory laparotomy. Spontaneous perforated viscus in patients undergoing PD is rare, but without prompt and aggressive intervention may be associated with significant morbidity and mortality. We discuss the case and review the literature highlighting the delay in the diagnoses of such cases and the role of imaging and exploratory laparotomy. Finally, recovery of multiple enteric pathogens in the workup of PD-associated peritonitis should raise the suspicion of possible viscus perforation.
\end{abstract}

Keywords: Peritonitis; Peritoneal dialysis; Viscus perforation

\section{Introduction}

In the treatment of end-stage renal disease (ESRD), peritoneal dialysis (PD) is a common therapeutic modality, but may be associated with complications. Some of the notable complications are outflow failure [1], pericatheter leakage [2], catheter cuff extrusion [3], intestinal perforation [3] and bleeding [4]. However, peritonitis still remains the most significant complication of PD [5] and is an important obstacle to long-term

Manuscript submitted May 31, 2018, accepted June 27, 2018

aDow Medical College, Karachi, Pakistan

bepartment of Internal Medicine, University of Toledo Medical Center, Toledo, OH, USA

'Department of Nephrology, University of Toledo Medical Center, Toledo, $\mathrm{OH}, \mathrm{USA}$

${ }^{\mathrm{d} D e p a r t m e n t}$ of Infectious Diseases, University of Toledo Medical Center, Toledo, $\mathrm{OH}, \mathrm{USA}$

'Corresponding Author: Joan Duggan, Department of Infectious Diseases, University of Toledo Medical Center, 3000 Arlington Ave., Toledo, OH 43614, USA. Email: Joan.duggan@utoledo.edu

doi: https://doi.org/10.14740/jmc3116w
PD therapy [6]. Some of the most common causes of peritonitis in PD patients are PD catheter exit site infection [7] and contamination during PD fluid exchange. In most cases, it is caused by a single organism while in $6-9 \%$ of patients, it is polymicrobial with multiple enteric organisms [8]. Gram positive bacteria constitute the most frequently isolated pathogens (45-65\%), followed by Gram negative bacteria $(25-40 \%)$ and fungi (3-6\%) [9-12]. Polymicrobial peritonitis should raise the suspicion of gastrointestinal pathology as the cause of peritonitis. It remains a diagnostic challenge to differentiate between peritonitis secondary to PD and peritonitis secondary to perforation as both entities may present with similar signs and symptoms. However, a delay in performing exploratory laparotomy in peritonitis secondary to perforation may result in adverse outcomes. We present the case of a PD patient with perforated diverticula which highlights some of the diagnostic difficulties encountered in this clinical setting.

\section{Case Report}

A 41-year-old female with Down's syndrome and ESRD secondary to IgA nephropathy was on PD after two failed renal transplants. She remained on immunosuppression because of her previous transplants. The patient presented with diffuse abdominal pain associated with fever and chills but was unable to give a significant history of the presenting illness due to her limited cognitive development. The physical exam on admission revealed diffuse abdominal pain and tenderness. Exam and laboratory findings (Table 1) were consistent with peritonitis. The computed tomography (CT) scan on admission revealed air in her peritoneal cavity which was thought to be from the PD catheter itself. She had initially received ceftazidime but was not responding well to guideline-directed therapy and was subsequently changed to vancomycin, meropenem and cefazolin after failure of initial therapy. The patient's condition rapidly deteriorated over the next $24 \mathrm{~h}$ and she became hypotensive with acute respiratory failure. She was intubated and transferred to the intensive care unit. On day 5, her PD culture grew Clostridium perfringens and Lactobacillus acidophilus which suggested possible gastrointestinal perforation. Surgical consultation was obtained and she was taken to the operating room for a diagnostic exploratory laparoscopy which revealed feculent fluid throughout her peritoneum along with extensive inflammation of her bowel and fibrinous exudate with particulate stool matter. An open laparotomy 
Table 1. Patient's Vitals and Laboratory Values

\begin{tabular}{|ll|}
\hline Vitals & \\
\hline Temperature & 102.5 \\
Blood Pressure & $143 / 90$ \\
\hline Pulse & 93 \\
\hline Respiration & 20 \\
\hline Laboratory values & \\
\hline White blood cell count & $11,300 \mathrm{cells} / \mathrm{mm}^{3}$ \\
\hline Hemoglobin & $8.6 \mathrm{~g} / \mathrm{dL}$ \\
\hline PD fluid white count & $9,420 \mathrm{cells} / \mathrm{mm}^{3}$ \\
\hline Cytology & Mixed inflammation, predominantly neutrophilic; proteinaceous material and negative for malignancy \\
\hline
\end{tabular}

was immediately performed and her sigmoid colon was found to be adhered to the retroperitoneum. A rigid sigmoidoscopy revealed a small perforation which was possibly diverticular in origin. A segmental colectomy was performed with colostomy formation. Her post-operative course was complicated by a perihepatic abscess that required interventional radiology guided drainage, and culture from the abscess revealed vancomycin resistant enterococci. The PD catheter culture also revealed Candida species and the patient was treated with ceftazidime, metronidazole, daptomycin, and fluconazole for 2 weeks. Unfortunately, she could not return to PD as a form of renal replacement therapy secondary to formation of intaabdominal adhesions subsequently required hemodialysis. She otherwise experienced a full recovery.

\section{Review of the literature}

Peritonitis is one of the major complications of PD [13]. This common complication is usually not associated with a perforation, and it can present a challenge in the timely diagnosis and management of an acute surgical abdomen. There have been few studies which discuss the presentation and differentiating factors of surgical abdominal emergencies in patients with PD compared to conventional PD associated peritonitis [14-16]. A review of the literature from inception of the PubMed database to March 2017 using the search terms peritoneal dialysis and viscus perforation revealed a total of 22 patients (Table 2) [14-16], out of which 13 (59\%) patients were males and nine (41\%) patients were females, with an average age of 62 years. There were eight (36.36\%) deaths in this review highlighting the high mortality rate in such cases. The most common cause of death in these patients was sepsis, which accounted for $37.5 \%$ of all deaths. Perforated diverticulitis was the cause of peritonitis in $45.45 \%$ of patients, followed by perforated appendicitis in $18 \%$ of patients. The most common organism recovered was $E$. coli, which was found in $59 \%$ of the cases. CT scans were done on six patients and none were diagnostic for perforation. Our findings highlight that the clinical picture is much more important in PD patients in the diagnosis of peritonitis than in non-PD patients, where CT scan is a very effective modality in the diagnosis of viscus perforation [17].

\section{Discussion}

The role of laparoscopy should also be considered in the diagnosis and treatment of peritonitis in PD patients in whom the suspicion for secondary cause of peritonitis is high. Eustace et al reported the first case diagnostic laparoscopy in a PD patient with acute appendicitis in 1996 [18] but the review suggested a reluctance to use laparoscopy early in the course of the disease for the diagnosis of secondary causes of peritonitis in patients not responding to appropriate therapy. Newer imaging modalities such as CT scan may be less useful in PD patients and heavy reliance on imaging may result in delayed diagnosis of perforation in PD patients [16]. Our review demonstrated a delay in the diagnosis of a perforation in this patient population ranging from 1 to 27 days with an average delay in diagnosis of 6 days [14-16]. Among the causes of reduced sensitivity of CT scanning in PD patients may be the use of povidone-iodine and antibiotic solutions for irrigation in bacterially contaminated peritoneum to reduce the incidence of abscess formation which is more easily detected radiologically [19]. There are several other reasons which can account for delay in diagnosis of perforation in PD patients. The most important reason is that most PD patients who have symptoms of peritonitis are more likely to have primary peritonitis related to the PD catheter and its use than having a perforated viscus. These patients are often started on empiric antibiotics intraperitoneally which provides partial treatment in cases of unsuspected perforated viscus, and may provide some symptomatic relief [16], leading to an erroneous sense of treatment response and delay in diagnosis of perforation. The amount of time it takes for a culture to reveal the organism also invariably adds to the delay in diagnosis. In the current case, there was a 5-day delay in the culture results of multiple enteric organisms which prompted the suspicion of gastrointestinal pathology. There may also be reluctance on behalf of the treating physician or surgeon to commit the PD patient to surgical exploration because of the possibility that adhesions secondary to surgery may render the peritoneal cavity unsuitable for continuous ambulatory peritoneal dialysis [16]. It is interesting to note that the two patients in Table 2 
Table 2. Characteristics of Patients on PD With Peritonitis From Perforation

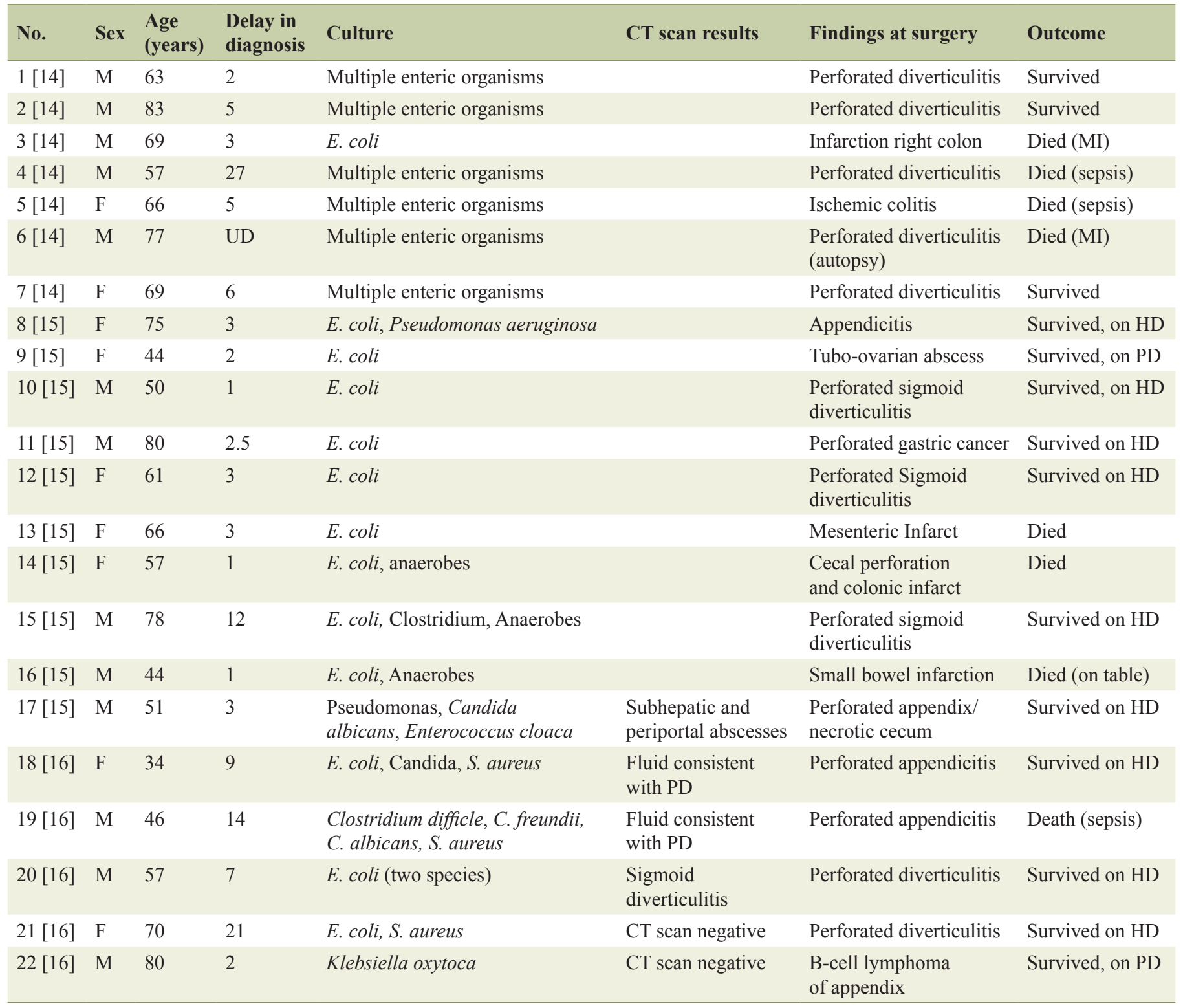

who did not have significant intra-abdominal pathology were able to continue on PD despite having laparotomy which may suggest that there may be other risk factors other than surgery that may render the peritoneal cavity unsuitable for PD after spontaneous rupture of the viscus.

\section{Conclusion}

The present case illustrates the challenges associated with the diagnosis of perforation in PD patients. To prevent delay, the clinician must pursue alternative diagnoses if there is no improvement or worsening of symptoms after $24-48 \mathrm{~h}$ from initiation of treatment. In addition, CT scans in PD patients may not be comparable to $\mathrm{CT}$ scans in non-PD patients with perforated viscus. Early exploration should be considered in patients who deteriorate or show little improvement of symptoms with antibiotic therapy in order to avoid significant morbidity and mortality.

\section{References}

1. Allon M, Soucie JM, Macon EJ. Complications with permanent peritoneal dialysis catheters: experience with 154 percutaneously placed catheters. Nephron. 1988;48(1):811.

2. Leblanc M, Ouimet D, Pichette V. Dialysate leaks in peritoneal dialysis. Semin Dial. 2001;14(1):50-54.

3. Fleisher AG, Kimmelstiel FM, Lattes CG, Miller RE. 
Surgical complications of peritoneal dialysis catheters. Am J Surg. 1985;149(6):726-729.

4. Mital S, Fried LF, Piraino B. Bleeding complications associated with peritoneal dialysis catheter insertion. Perit Dial Int. 2004;24(5):478-480.

5. Mactier R. Peritonitis is still the achilles' heel of peritoneal dialysis. Perit Dial Int. 2009;29(3):262-266.

6. Mizuno M, Ito Y, Tanaka A, Suzuki Y, Hiramatsu H, Watanabe M, Tsuruta Y, et al. Peritonitis is still an important factor for withdrawal from peritoneal dialysis therapy in the Tokai area of Japan. Clin Exp Nephrol. 2011;15(5):727-737.

7. van Diepen AT, Tomlinson GA, Jassal SV. The association between exit site infection and subsequent peritonitis among peritoneal dialysis patients. Clin J Am Soc Nephrol. 2012;7(8):1266-1271.

8. Holley JL, Bernardini J, Piraino B. Polymicrobial peritonitis in patients on continuous peritoneal dialysis. Am J Kidney Dis. 1992;19(2):162-166.

9. Ghali JR, Bannister KM, Brown FG, Rosman JB, Wiggins KJ, Johnson DW, McDonald SP. Microbiology and outcomes of peritonitis in Australian peritoneal dialysis patients. Perit Dial Int. 2011;31(6):651-662.

10. Mujais S. Microbiology and outcomes of peritonitis in North America. Kidney Int Suppl. 2006;103:S55-62.

11. Zelenitsky S, Barns L, Findlay I, Alfa M, Ariano R, Fine A, Harding G. Analysis of microbiological trends in peritoneal dialysis-related peritonitis from 1991 to 1998. Am J Kidney Dis. 2000;36(5):1009-1013.
12. Szeto CC, Kwan BC, Chow KM, Lau MF, Law MC, Chung KY, Leung CB, et al. Coagulase negative staphylococcal peritonitis in peritoneal dialysis patients: review of 232 consecutive cases. Clin J Am Soc Nephrol. 2008;3(1):91-97.

13. Perez Fontan M, Rodriguez-Carmona A, Garcia-Naveiro R, Rosales M, Villaverde P, Valdes F. Peritonitis-related mortality in patients undergoing chronic peritoneal dialysis. Perit Dial Int. 2005;25(3):274-284.

14. Wellington JL, Rody K. Acute abdominal emergencies in patients on long-term ambulatory peritoneal dialysis. Can J Surg. 1993;36(6):522-524.

15. Miller GV, Bhandari S, Brownjohn AM, Turney JH, Benson EA. 'Surgical' peritonitis in the CAPD patient. Ann R Coll Surg Engl. 1998;80(1):36-39.

16. Carmeci C, Muldowney W, Mazbar SA, Bloom R. Emergency laparotomy in patients on continuous ambulatory peritoneal dialysis. Am Surg. 2001;67(7):615-618.

17. Saklayen MG. CAPD peritonitis. Incidence, pathogens, diagnosis, and management. Med Clin North Am. 1990;74(4):997-1010.

18. Eustace J, Lappin D, Keane F, Mellotte GJ, Keogh JA. Laparoscopic-aided diagnosis of recurrent peritonitis in a patient on CAPD. Nephrol Dial Transplant. 1996;11(11):2338-2339.

19. Sindelar WF, Mason GR. Intraperitoneal irrigation with povidone-iodine solution for the prevention of intra-abdominal abscesses in the bacterially contaminated abdomen. Surg Gynecol Obstet. 1979;148(3):409-411. 